\title{
Zur Biologie der Kieselsäure und der Tonerde in den Vogelfedern. \\ Von
}

Dr. Max Gonnermann.

(Aus dem Institut für Pharmakol. und physiol. Chemie 2u Rostock.)

(Der Redaktion zugegangen am 21. März 1918.)

Aus dem Auftreten der Kieselsäure und Tonerde in Organen von Tieren und Menschen, worüber ich eine Untersuchung in dieser Zeitschrift, Bd. 99 und eine zweite anderweitig veröffentlicht habe, war leicht $z u$ folgern, da $B$ auch in den Vogelfedern beide Substanzen sich vorfinden könnten. Ich habe demgemä $B$ eine Reihe Federarten untersucht. Besonders regten die Resultate von Drechsel und Winogrodow, wonach in den Vogelfedern der Orthokieselsäureester $\mathrm{Si}\left(\mathrm{OC}_{34} \mathrm{H}_{59} \mathrm{O}\right)_{4}$ eines zweiwertigen Alkohols: $\mathrm{C}_{34} \mathrm{H}_{60} \mathrm{O}_{2}$ enthalten ist, dazu an. Carl Cerny ${ }^{1}$ ) wiederholte die Versuche der genannten beiden Forscher und stellte diese Substanz sowohl aus Gänsefedern, als aus Hühnerfedern dar. Die Federstämme der Gans lieferten $0,17 \%$, die Fahnen 0,15\% dieser Substanz. Hühnerfedern aber lieferten $0,346 \%$, also das Doppelte. Der Gehalt an Kieselsäure, der bei dieser Substanz nach Drechsel 2,75\% betragen mußte, betrug aber in maximo $0,4 \%$ und war mehrmals Null! Offenbar liegt ein Gemisch von Fettsäureestern einer oder mehrerer hochmolekularen Alkohole vor, die dem Bürzeldrüsensekret entstammen, und das Vorkommen von Kieselsäure in den Federn ist nach Cerny nur ein zufälliges, eine Verunreinigung durch Kieselstaub, eine Ansicht, welche Kobert stets bekämpft hat, denn auch

1) Carl Cerný, Zur Frage des Vorkommens von Kieselsäure im Organismus. Diese Zeitschr., Bd. 62, S. 296 (1909). 
aus dem Ätherextrakt des mit Alkohol vorher entwässerten Vogelblutes (Gänse, Hühner, Tauben), sowie aus der Gänseleber konnte Cerny eine kieselsäurehaltige Substanz mit $0,05-0,8 \%$ Kieselsäure isolieren.

Auf diese interessanten Versuche hin schien es mir dankenswert, eine Reihe Aschenanalysen möglich reiner Vogelfedern anzustellen. Es war zu hoffen, daß dadurch die Frage des Vorkommens der Kieselsäure in den einzelnen Teilen der Federn geklärt werden würde.

Es wurden nur die Flügelfedern verarbeitet und zwar von den größeren Federn Kiele und. Fahnen gesondert, von den kleinen Schwungfedern Kiele und Fahnen zusammen, und zwar auf Kieselsäure und auf Tonerde. Das Material wurde im Porzellantiegel verbrannt, aus der Asche die Kieselsäure nach der gewöhnlichen analytișchen Methode abgeschieden und sodann durch Schmelzen mit Ammoniumfluorid als Verlust bestimmt. Die Tonerde wurde im Filtrat der ausgelaugten Asche gesucht und bestimmt. (Siehe Tabellen.)

Gorup-Besanez fand in der Asche fleischfressender $27 \%$, von Fischen lebender nur 10,5\%, dagegen in den Federn körnerfressender Vögel $40 \%$ Kieselsäure, und schließt aus diesen Befunden mit Recht auf einen Einfluß der Nahrung auf den Kieselsäuregehalt der Federn. Dazu stimmt, daß er in den Federn ganz junger Vögel den Gehalt an Kieselsäure geringer fand. Analog den Vogelfedern sind auch die Igelstacheln stets kieselsäurehaltig, wie denn weiter analog diesen Befunden auch die Haare von Tieren und Menschen diese Substanz enthalten. So fand Gorup-Besanez in der Asche von Rehhaaren $8,1 \%$, Schafwolle $8,3 \%$, Bockhaaren 9,4\%, Meerschweinchenhaaren $11,8 \%$, Hundehaaren $12,5 \%$, Pferdehaaren 14,6\%. Kieselsäure. Im Haupthaar fand Baudrimont im schwarzen $6,611 \%$, im weißen $12,3 \%$, im braunen $30,6 \%$, im blonden $30,7 \%$, im roten $42,5 \%$ der Asche an Kieselsäure. Gorup-Besane z fand in der Asche von weißem Haar 9,5\%, im braunen $13,9 \%$. In den Haaren von Neugeborenen fällt der Prozentsatz natürlich bedeutend $a b$ auf $0,083-0,103$, und steigt mit dem Alter, sodaß beim zwei- 
Max Gonnermann,

\begin{tabular}{|c|c|c|c|c|c|c|c|c|c|}
\hline & & 1 & 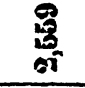 & 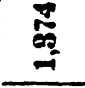 & $\frac{5}{\infty}$ & 店 & $\frac{7}{5}$ & $\begin{array}{l}\mathscr{8} \\
\mathbb{\infty} \\
\infty\end{array}$ & 1 \\
\hline & $\underset{0}{0}$ & 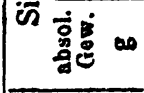 & :̊ํํ & $\begin{array}{l}8 \\
8 \\
0\end{array}$ & $\frac{10}{0}$ & 䏰 & 8̊ & 芯 & 1 \\
\hline & $\stackrel{0}{\bullet}$ & 兽 & 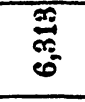 & कू & $\begin{array}{l}\text { कृ } \\
\text { o. } \\
\text { ज. }\end{array}$ & $\stackrel{\infty}{\stackrel{\infty}{a-j}}$ & $\underset{-1}{\infty}$ & 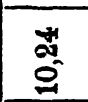 & 1 \\
\hline & is & مه & 용 & $\stackrel{\circ}{\circ}$ & 递 & 요 & $\stackrel{2}{\circ}$ & 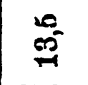 & 1 \\
\hline & & 兽 & $\tilde{\sigma}$ & : & 象 & 疍 & 0 & $\frac{\overrightarrow{5}}{3 j}$ & 1 \\
\hline & 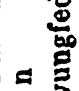 & 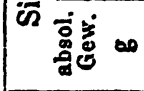 & ठृ. & $\begin{array}{l}8 \\
8 \\
0\end{array}$ & ᄋ్ & $\frac{5}{0}$ & 0 & $\overline{8}$ & 1 \\
\hline $\begin{array}{l}\text { : } \\
\text { జ } \\
0\end{array}$ & 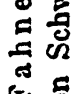 & 造 은 & 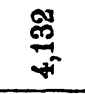 & $\rightarrow$ & $\stackrel{0}{0}$ & 尔 & 8 & $\stackrel{0}{\stackrel{0}{\alpha}}$ & 1 \\
\hline$\stackrel{\infty}{2}$ & 递 & के & $\infty_{\infty}^{\infty}$ & 요 & $\stackrel{0}{\rightarrow}$ & $\stackrel{0}{\rightarrow}$ & $\stackrel{0}{=}$ & $\stackrel{0}{\circ}$ & 1 \\
\hline$\Xi$ & गु & స్ & 怘 & $\stackrel{0}{=}$ & กึ & $\stackrel{\Im}{\sim}$ & શ & $\stackrel{0}{=}$ & 1 \\
\hline జี & & 兽 & 동 & 离 & 㿣 & ह & 0 & क्ञ & 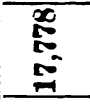 \\
\hline 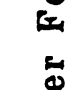 & 总 & 朋宮高 & O্ & $\frac{0}{0}$ & ㅎ․ & ஜ̊ & 0 & $\overline{8}$ & $\begin{array}{l}\infty \\
8 \\
0 \\
0\end{array}$ \\
\hline $\begin{array}{l}\sigma \\
\sigma\end{array}$ & \begin{tabular}{l}
0 \\
0 \\
0 \\
\hdashline
\end{tabular} & 总 & $\underset{\Xi}{=}$ & $\begin{array}{l}\stackrel{\$}{\infty} \\
\text { ळ. } \\
0\end{array}$ & $\frac{10}{20}$ & $\frac{\pi}{0}$ & है & $\begin{array}{l}5 \\
\infty \\
\infty \\
0\end{array}$ & $\begin{array}{l}\infty \\
\stackrel{0}{0} \\
\text { mi }\end{array}$ \\
\hline की & ङ્仓 & . & $\stackrel{0}{0}$ & $\stackrel{0}{=}$ & 오 & 요 & ㅇ. & 요 & 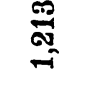 \\
\hline & هั & $\frac{7}{\text { กี }}$ & : & $\stackrel{\circ}{=}$ & నొ & ฯ & $\underset{\sim}{-}$ & $\stackrel{\oplus}{-}$ & 읔 몷 \\
\hline & & 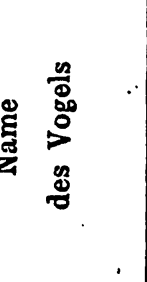 & 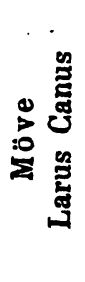 & 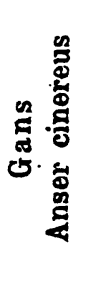 & 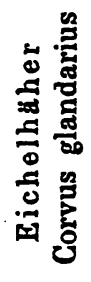 & 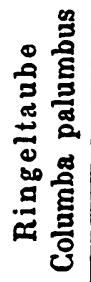 & 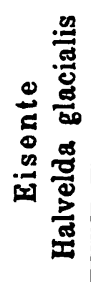 & 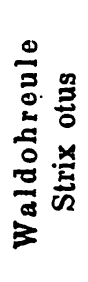 & 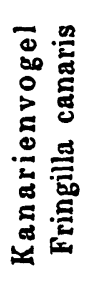 \\
\hline & & 点 & ن- & ல่ & هి & ن & $20^{\circ}$ & $\varphi^{\circ}$ & $N$ \\
\hline
\end{tabular}


Zur Biologie der Kieselsäure und der Tonerde in den Vogelfedern. $\quad 81$

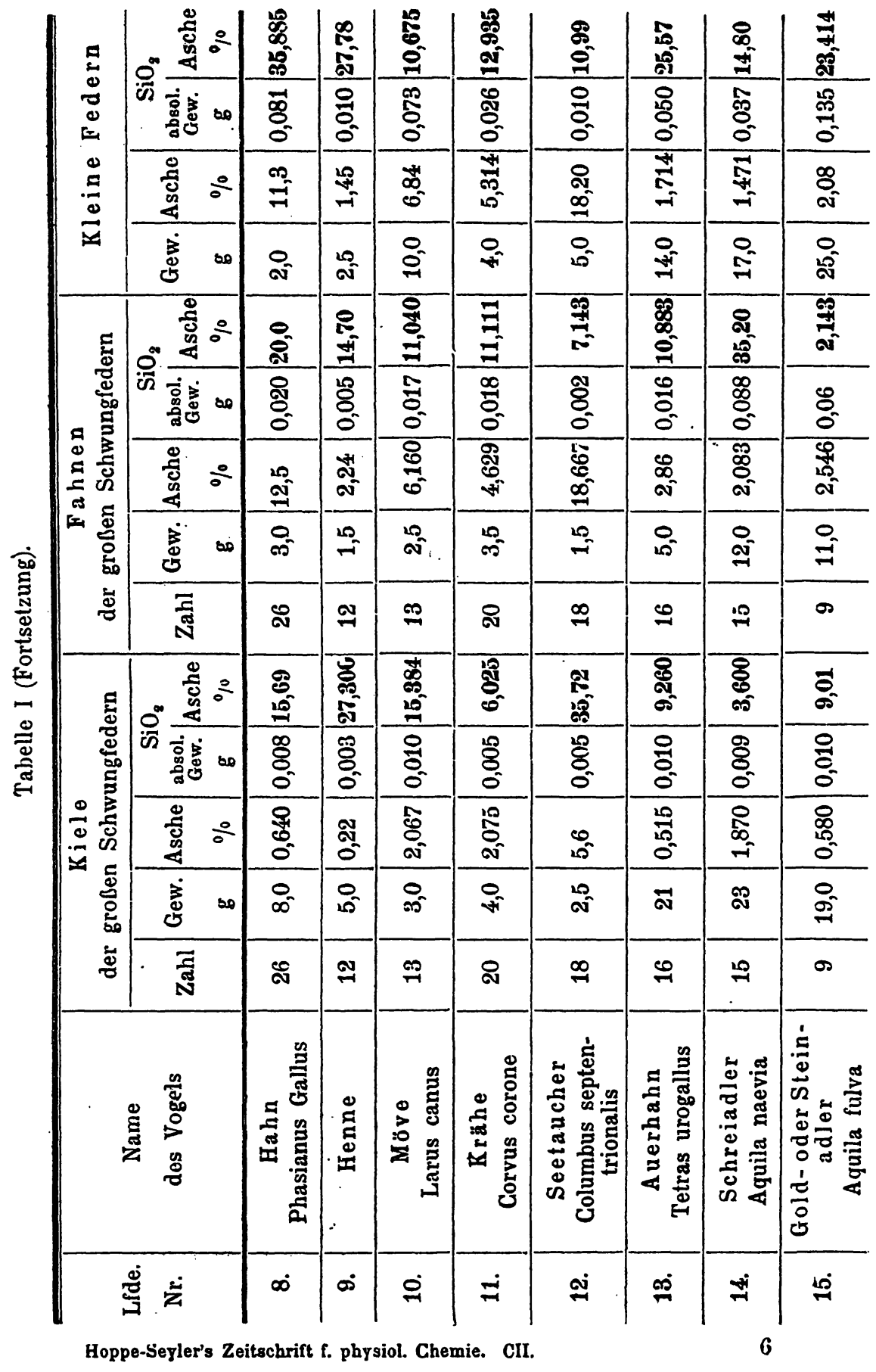


Tabelle II.

\begin{tabular}{|c|c|c|}
\hline $\begin{array}{l}\text { Lrde. } \\
\text { Nr. }\end{array}$ & $\begin{array}{c}\text { Name } \\
\text { des Vogels }\end{array}$ & Aschenbestandteile auf Federn berechnet \\
\hline 1. & $\begin{array}{l}\text { Möve } \\
\text { Larus canus }\end{array}$ & $\begin{array}{l}\text { Asche enthält Spuren von Bisenoxyd, keine } \\
\text { Tonerde. }\end{array}$ \\
\hline 2. & $\begin{array}{l}\text { Gans } \\
\text { Anser cinereus }\end{array}$ & Asche frei von Tonerde. \\
\hline 3. & $\begin{array}{l}\text { Eichelhäher } \\
\text { Corvus glanda- } \\
\text { rius }\end{array}$ & $\begin{array}{l}\text { Asche enthält } 0,882 \% \text { Eisenoxyd, } 2,46 \% \text { Ton- } \\
\text { erde der Kiele; Fahnen and kleine Federn } \\
\text { gleichfalls, jedoch wenig. }\end{array}$ \\
\hline 4. & $\begin{array}{c}\text { Ringeltaube } \\
\text { Columba palum- } \\
\text { bus }\end{array}$ & $\begin{array}{l}\text { Tonerde war in den Federn, wenn auch nicht } \\
\text { wägbar vorhanden; ebenfalls Eisenoxyd. }\end{array}$ \\
\hline 5. & $\begin{array}{c}\text { Eis en te } \\
\text { Halvelda glacialis }\end{array}$ & $\begin{array}{l}\text { Große Schwungfedern sind frei von Tonerde, da- } \\
\text { gegen findet sich solche in den kleinen Federn. }\end{array}$ \\
\hline 6. & $\begin{array}{l}\text { Waldohreule } \\
\text { Strix otus }\end{array}$ & $\begin{array}{l}\text { Die Kiele enthalten } 0,234 \% \text { Tonerde und Eisen- } \\
\text { oxyd; die Fahnen 1,41\%, die kleinen Federn } \\
0,369 \% \text { davon. }\end{array}$ \\
\hline 7. & \begin{tabular}{|c|} 
Kanarien- \\
vogel \\
Fringilla canaris
\end{tabular} & Tonerdegehalt $1,984 \%$ für sämtliche Federn. \\
\hline 8. & $\begin{array}{c}\text { Hahn } \\
\text { Phasianus Gallus }\end{array}$ & $\begin{array}{l}\text { Kiele sind frei von Tonerde, Fahnen und kleine } \\
\text { Federn nur wenig. }\end{array}$ \\
\hline 9. & Henne & Wie bei dem Hahn. \\
\hline 10. & $\begin{array}{l}\text { Möve } \\
\text { Larus canus }\end{array}$ & 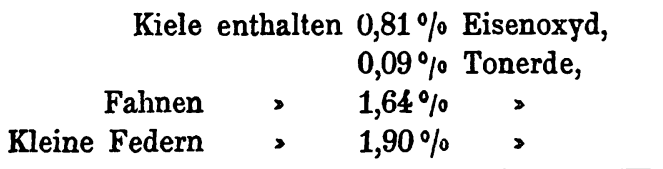 \\
\hline 11. & $\begin{array}{l}\text { Krähe } \\
\text { Corvus corone }\end{array}$ & $\begin{array}{l}\text { Kiele enthalten eine geringe Menge Tonerde; } \\
\text { Fahnen wenig Tonerde und Eisenoxyd; } \\
\text { kleine Federn gleichfalls nur sehr geringe } \\
\text { Mengen. }\end{array}$ \\
\hline 12. & $\begin{array}{c}\text { Seetaucher } \\
\text { Columbus septen. } \\
\text { trionalis }\end{array}$ & $\begin{array}{l}\text { Kiele und Fahnen enthalten wohl Tonerde, } \\
\text { jedoch wegen des geringen Materials nur } \\
\text { Spuren. Auch die kleinen Federn enthalten } \\
\text { : sehr wenig. }\end{array}$ \\
\hline
\end{tabular}


Tab. II (Fortsetzung).

\begin{tabular}{|c|c|c|}
\hline $\begin{array}{l}\text { Lfde. } \\
\text { Nr. }\end{array}$ & $\begin{array}{l}\text { Name } \\
\text { des Vogels }\end{array}$ & Aschenbestandteile auf Federn berechnet \\
\hline 13. & $\begin{array}{l}\text { Auerhahn } \\
\text { Tetrao urogallus }\end{array}$ & $\begin{array}{l}\text { Kiele enthalten nur wenige Milligramme; Fahnen } \\
\text { und kleine Federn nicht wägbare Mengen. }\end{array}$ \\
\hline 14. & $\begin{array}{l}\text { Schreiadler } \\
\text { Aquila nawia }\end{array}$ & $\begin{array}{l}\text { In den Kielen fanden sich } \\
0,183 \% \text { Tonerde, } \\
0,150 \% \text { Eisenoxyd, } \\
\text { in den Fahnen } 2,14 \% \text { Tonerde, } \\
5,36 \% \text { Eisenoxyd, } \\
\text { in den kleinen Federn } \\
0,212 \% \text { Tonerde, } \\
0,241 \% \text { Eisenoxyd. }\end{array}$ \\
\hline 15. & $\begin{array}{l}\text { Gold- } \\
\text { od. Steinadler } \\
\text { Aquila fulva }\end{array}$ & $\begin{array}{r}\text { Kiele enthalten nur wenig Tonerde, } \\
\text { Fahnen } \\
\begin{aligned} 1,143 \% & \text { Tonerde, } \\
& 0,643 \%\end{aligned} \text { Eisenoxyd, } \\
\text { kleine Federn } 0,472 \% \text { T'onerde, } \\
0,236 \% \text { Eisenoxyd. }\end{array}$ \\
\hline
\end{tabular}

jährigen blonden Kind 0,115\%, bei einem 17 jährigen $0,151 \%$ vorhanden sind. Ein dunkelbraunes Haupthaar eines 59jährigen Mannes enthielt $0,233 \%$, die grauen Haare eines 63 jährigen allerdings nur noch 0,100 , und die eines 65 jährigen $0,098 \%$. Doch gelten diese niedrigen Zahlen für Haare und nicht für Asche; so dürfte sich z. B. der Aschengehalt der genannten 15 jährigen auf $18,4 \%$ berechnen.

Aus meinen früheren Untersuchungen will ich vergleichend nur hervorheben, daß sich in der Asche von

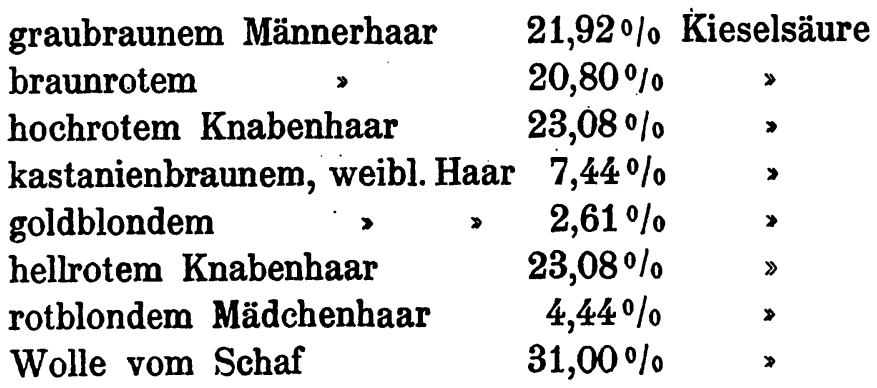

fanden. 
Die Federn sind ein epitheliales Gebilde wie die Haare. Daher kann es uns nicht wundern, wenn ich durchweg auch in den Federn Kieselsäure fand und $z$ war in den Fahnen der großen Schwungfedern der Ringeltaube bis zu 77\% der Asche. Diese Kieselsäure stammt aus derjenigen der Pflanzennahrung, wird also von den Tieren resorbiert, durchwandert den ganzen Organismus und gelangt in den Federn zur Ausscheidung, wobei sie deren Resistenz vermehrt. Da bei anderen Vögeln die Menge viel geringer ist, so ist daraus zu schließen, daß wohl keine bestimmte organische chemische Verbindung mit feststehendem Siliciumgehalt vorliegt, sondern da $B$ nach dem zufälligen Gehalt des Organișmus bezw. des Futters an Kieselsäure bald mehr, bald weniger davon in den Federn zur Ablagerung kommt. Vielleicht gilt dasselbe auch für die Tonerde. Immerhin ist das Vorkommen der Tonerde in den Federn doch bemerkenswert.

Ich habe auf Seite 80-83 zwei Tabellen gegeben, deren erste sich auf Kieselsäure bezieht, während die zweite es mit der Tonerde zu tun hat. Bei der Kieselsäure habe ich eine Dreiteilung des Untersuchungsmateriales eintreten lassen nach Kielen, Fahnen und kleinen Federn. 